\title{
Neutrophil/Lymphocyte Ratio, Platelet/Lymphocyte Ratio, and Mean Platelet Volume for Detection of Resectable Pancreas Cancer
}

\author{
Kemal Turker Ulutas, Inanc Samil Sarici and \\ Ozgul Duzgun \\ Additional information is available at the end of the chapter \\ http://dx.doi.org/10.5772/intechopen.76168
}

\begin{abstract}
Several biomarkers have been preferred for the early diagnosis of pancreatic adenocancer (PAC), but most are not ready to be included as part of the routine diagnostic algorithm because they still lack sensitivity, specificity or reproducibility. CA19-9 is the most widely used serum-based marker for the diagnosis and follow-up of pancreatic cancer. However, CA19-9 lacks sensitivity for early or small-diameter pancreatic cancers. For more than 3 decades, information on neutrophil/lymphocyte ratio (NLR), platelet/lymphocyte ratio (PLR), mean platelet volume (MPV) has been widely available to health care practitioners, as part of the data provided in the full blood count. However, these biomarkers have more than used in the routine. The present chapter shares the prognostic significance of the hematological parameters in the light of our own findings and recent studies in the literature.
\end{abstract}

Keywords: NLR, PLR, MPV, resectable pancreas cancer, biomarker

\section{Introduction}

Pancreatic adenocarcinoma is a devastating disease with an extremely poor prognosis and prompt diagnostic evaluation is vital when PAC is suspected. CA19-9 is the most widely used serum-based marker for the diagnosis and follow-up of pancreatic cancer [1]. The diagnostic role of CA19-9 as a test for the detection of pancreatic malignancy remains poorly defined, because, as in other diagnostic modalities, the utility of CA19-9 has several 
confounding limitations. The sensitivity and specificity of CA19-9 vary, ranging from 70 to $90 \%$ and 68 to $91 \%$, respectively. However, CA19-9 lacks sensitivity for early or smalldiameter pancreatic cancers. Poorly differentiated pancreatic cancers also appear to produce less CA19-9 than either moderately or well-differentiated cancers. Another limitation is that CA19-9 can also be elevated in benign inflammatory and cholestatic diseases of the pancreaticobiliary tract $[2,3]$.

After inflammatory processes have emerged as key mediators of pancreatic cancer development and progression, many inflammatory pathways have been identified in recent years. Neutrophil/lymphocyte ratio (NLR), platelet/lymphocyte ratio (PLR), and mean platelet volume (MPV) are the most used in the literature [4-6]. Elevated NLR has reportedly been associated with poor survival following resection or chemotherapy in a variety of cancer. In colorectal cancer, an increasing number of studies have reported an association between elevated NLR and poor prognosis [7]. The first study done by us, including a total of 41 resectable PAC patients and 43 age-matched and sex-matched healthy participants [8]. NLR, PLR, and MPV were significantly higher in preoperative stage 1 and stage 2 PAC patients compared with age-matched and sex matched healthy participants (5.51 vs. $2.5, \mathrm{P}=0.002 ; 180$ vs. $134, \mathrm{P}=0.017 ; 9.2$ vs. $2.5 \mathrm{fl}, \mathrm{P}=0.004$ ) (Table 1). Our results suggested that NLR, PLR and MPV might be used as easily available additional biomarkers for PAC in screening general population (Figure 1).

The role of new tumor marker PLR has been defined recently in the prognosis of PAC [9]. Miglani et al. reported that PLR has been at least as good as CA 19-9 as diagnostic marker to differentiate between malignant and inflammatory head mass of pancreas. This is based on the fact that PAC causes thrombosis and lymphocytopenia. Platelet activation is a link in the pathophysiology of diseases prone to thrombosis and inflammation. Lymphocytopenia occurs due to systemic inflammation caused by cancers that release a number of inhibitory immunologic mediators [10]. The diagnostic value of platelet size has recently been shown to be elevated in neoplastic disorders particularly in gastric cancer. Moreover, it has been determined that platelet size has a predictive value for bone marrow metastasis in patients with solid tumors [11]. Numerous platelet markers, including MPV, have been investigated in connection with both thrombosis and inflammation.

\begin{tabular}{llll}
\hline & PAC & Control & P value \\
\hline Neutrophil & $6092 \pm 4212$ & $3944 \pm 1219$ & 0.002 \\
PLT & $230 \pm 84$ & $244 \pm 64$ & 0.39 \\
MPV & $9.21 \pm 1.2$ & $8.5 \pm 0.82$ & 0.004 \\
NLR & $5.51 \pm 7.3$ & $2.5 \pm 1.1$ & 0.002 \\
PLR & $180 \pm 103$ & $135 \pm 65$ & 0.017 \\
\hline
\end{tabular}

Abbreviations: PLT: platelet; MPV: mean platelet volume; NLR: neutrophil/lymphocyte ratio; PLR: platelet/lymphocyte ratio; PAC: pancreatic cancer

Table 1. Hematological results of the patients and controls. 


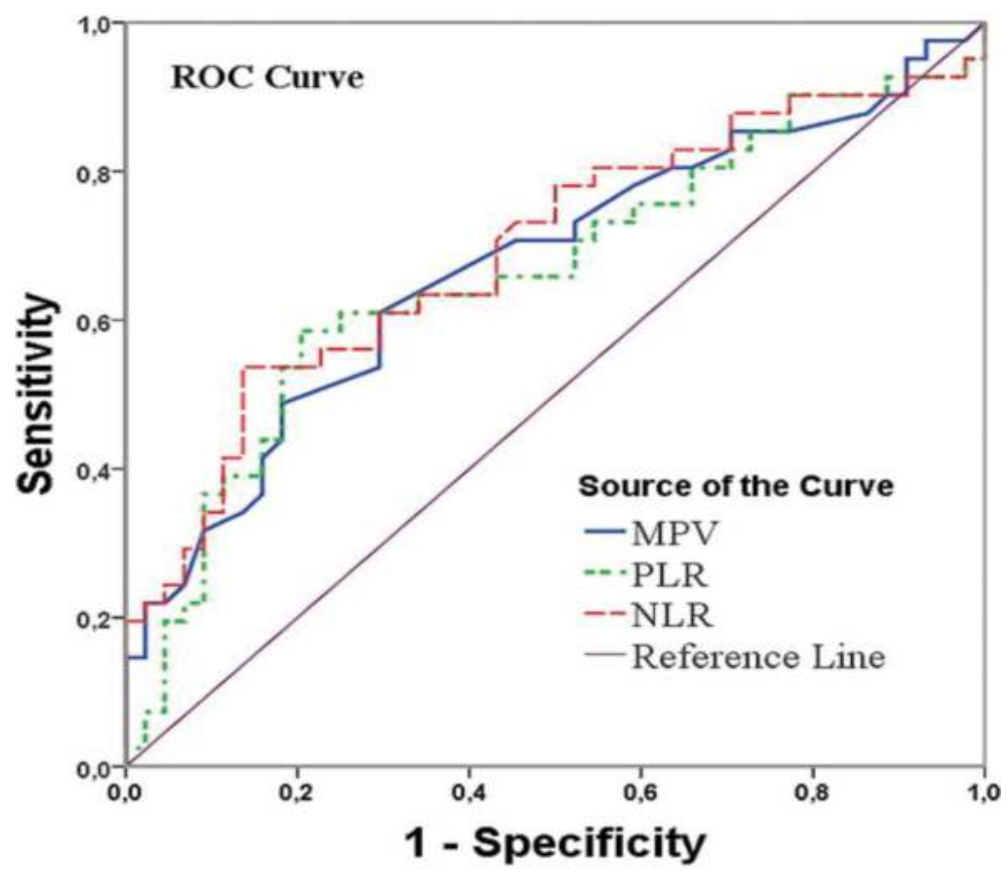

Figure 1. Receiver operating characteristic curves NLR, PLR and MPV for predicting stage 1 and stage 2 pancreatic cancer. Abbreviations: MPV: mean platelet volume; NLR: neutrophil/lymphocyte ratio; PLR: platelet/lymphocyte ratio.

\section{Inflammation and PAC}

In the development and progression of cancer, inflammation is a crucial and essential process [12]. Persistence of the inflammatory process within the tumor leads to an increase in the proliferation of tumor cells, angiogenesis, and the inhibition of apoptosis [13, 14]. Several reports have suggested that markers of systemic inflammation including cytokines, C-reactive protein, NLR, and PLR may provide useful information on the prognosis of colorectal gastrointestinal cancer $[15,16]$. Thus, pathogenesis of PAC appears to be an inflammation-driven malignancy, as well as colorectal gastrointestinal cancer. Usually, cancer cells are a source of inflammatory cytokines and growth factors. Interleukin-6 (IL-6) is an inflammatory cytokine that can cause carcinogenesis through several signal pathways involved in carcinogenesis, as well as metastasis of a variety of malignancies, including PAC [17].

It has been shown that PAC patients have higher levels of IL-6 compared with a healthy control group [18]. We acknowledged that IL-6 is released from leukocytes and is also able to activate the production of IL-6 by tumor cells through the IL-6 receptor. Besides their role in homeostasis, platelets and leucocytes take part in the pathophysiology of tumor angiogenesis [19]. Platelets are known to be the major transporter of vascular endothelial growth factor, which is the target for antiangiogenic therapies. Vascular endothelial growth factor accelerates the formation of blood vessels in the tumor and facilitates infiltration and spread to 
adjacent tissues, which in turn promotes the formation of metastases [20]. Solid tumors such as renal, gastric, and colon malignancies produce IL-6, which induces the proliferation and differentiation of megakaryocyte progenitors through specific receptors. This process causes platelet activation and aggregation. Platelet size has been shown to reflect changes in the level of platelet stimulation and the rate of platelet production.

According to the literature, lymphocytes play a key role in cytotoxic cell death and the production of cytokines that inhibit proliferation and metastatic spread of tumor cells. In contrast, neutrophils have a protumor effect by being the primary source of circulating angiogenesisregulating chemokines, growth factors, and proteases [4]. Elevated neutrophil levels may result in an increase in angiogenesis, which promotes development and progression of the neoplasm [6]. Therefore, NLR can be considered as the balance between protumor inflammatory status and antitumor immune status. At present, there is little information on the relevance of these prognostic markers to both diagnosis and monitoring of PAC. Similarly as being in our experience, newly diagnosed PAC patients have high NLR and PLR values than healthy human.

\section{Diagnostic weakness and missing points}

Even with decades passed, measurement of this parameter is still not standardized, as it can easily be obtained with electronic meters. This is a major flaw because many pre-analytical and analytical variables can affect platelet size. The pre-analytical variables include vascular occlusion method, the correctness of the filling of the vial and the mixing of the sample, the type of anticoagulant, the storage temperature and the duration of the analysis. Any inflammatory or malignant process can lead to an increase in these parameters [21].

In practice, these markers, if used alone, may have a low positive predictive value in screening an asymptomatic population. Getting in touch with EDTA, ethylene diamine tetra acetic acid, the most common anticoagulant used in laboratory practice, effects the platelet morphology and leads to swelling and an increase in volumes. The differences in the methodology of platelet counting with different automated analytics are most like to be major analytical variable for the measurement [22].

The poor standardization of the number of physiological variables affecting platelet size and the poor standardization of this parameter makes it very unlikely that small differences in this parameter, defined by clinical trials in various clinical conditions, could be used for clinical purposes. In the future, better methodological standardization and more personalized reference intervals may make them as a reliable parameter for differential diagnosis and prognostic identification in daily clinical practice, but there is a need for well-designed clinical trials to confirm this hypothesis [23].

\section{Diagnostic efficiency and strengths}

Certainly, the most important advantage is their cost-efficiency. In routine analyzes of PAC, several parameters have been being used at high cost. These parameters have so low cost 
which cannot be easily overlooked. Additionally, we speculate that increased MPV in a patient group newly diagnosed with PAC may be a reflection of ongoing inflammation, and it can be related to increased levels of cytokines, particularly IL-6. Thus, we suggest that MPV could be used for detection of PAC instead of CA19-9. Increased MPV value, an indicator of platelet volume, points the presence of a subpopulation of young, metabolically and enzymatically more active platelets taking part in the process of homeostasis. NLR and PLR are two representative indices of systemic inflammation [24]. It has been shown that a preoperative NLR of greater than 4 or 5 is associated with a poor outcome in gastric cancer, non-small-cell lung cancer, and ovarian cancer. Neutrophils and leukocytes play a crucial role in the host systemic inflammatory response. A nonspecific systemic inflammatory response due to a tumor leads to an increase in the levels of circulating neutrophils and an elevated NLR, all of which are also clearly demonstrated in our study.

\section{Conclusion}

As in our results and the literature, the patients with PAC have higher levels of these biomarkers than healthy people. Thus, patients with high NLR, PLR, and MPV with suspicious symptoms and/or signs of PAC are candidates for early evaluation, which can prevent delay in the diagnosis of PAC. Therefore, prospective studies with inflammatory marker screening as IL-6, TNF on a larger number of asymptomatic patients are needed to compare the performance of NLR, PLR, and MPV with that of other diagnostic and monitoring tests to confirm their diagnostic utility.

\section{Author details}

Kemal Turker Ulutas ${ }^{1}$, Inanc Samil Sarici ${ }^{2 *}$ and Ozgul Duzgun ${ }^{3}$

*Address all correspondence to: issarici2015@gmail.com

1 Department of Clinical Biochemistry, Antakya State Hospital, Hatay, Turkey

2 Department of General Surgery, Kanuni Sultan Suleyman Training and Research Hospital, Istanbul, Turkey

3 Department of Surgical Oncology, Umraniye Training and Research Hospital, Istanbul, Turkey

\section{References}

[1] Zhang GM, Bai SM, Zhang GM, Ma XB. Reference intervals of carbohydrate antigen 19-9 in the apparently healthy adult population. Journal of Clinical Laboratory Analysis. 2018;e22380. https://doi.org/10.1002/jcla.22380 
[2] Bedi MMS, Gandhi MD, Jacob G, Lekha V, Venugopal A, Ramesh H. CA 19-9 to differentiate benign and malignant masses in chronic pancreatitis: Is there any benefit? Indian Journal of Gastroenterology. 2009;28(1):24-27

[3] Goonetilleke KS, Siriwardena AK. Systematic review of carbohydrate antigen (CA 19-9) as a biochemical marker in the diagnosis of pancreatic cancer. European Journal of Surgical Oncology (EJSO). 2007;33(3):266-270

[4] Gaitanidis A, Patel D, Nilubol N, Tirosh A, Sadowski S, Kebebew E. Markers of systemic inflammatory response are prognostic factors in patients with pancreatic neuroendocrine tumors (PNETs): A prospective analysis. Annals of Surgical Oncology. 2018; 25(1):122-130

[5] Mannaerts D, Heyvaert S, De Cordt C, Macken C, Loos C, Jacquemyn Y. Are neutrophil/lymphocyte ratio (NLR), platelet/lymphocyte ratio (PLR), and/or mean platelet volume (MPV) clinically useful as predictive parameters for preeclampsia? The Journal of Maternal-Fetal \& Neonatal Medicine: the Official Journal of the European Association of Perinatal Medicine, the Federation of Asia and Oceania Perinatal Societies, the International Society of Perinatal Obstet. 2017:1-8. DOI: 10.1080/14767058.2017.1410701

[6] Rejec A, Butinar J, Gawor J, Petelin M. Evaluation of complete blood count indices (NLR, PLR, MPV/PLT, and PLCRi) in healthy dogs, dogs with periodontitis, and dogs with oropharyngeal tumors as potential biomarkers of systemic inflammatory response. Journal of Veterinary Dentistry. 2017;34(4):231-240

[7] Zhou WW, Chu YP, An GY. Significant difference of neutrophil-lymphocyte ratio between colorectal cancer, adenomatous polyp and healthy people. European Review for Medical and Pharmacological Sciences. 2017;21(23):5386-5391

[8] Ulutas KT, Sarici IS. Could neutrophil/lymphocyte ratio, platelet/lymphocyte ratio, and mean platelet volume serve as potential biomarkers for detection of resectable pancreas ca? International Journal of Clinical and Experimental Medicine. 2016;9(6):11865-11870

[9] Miglani RK, Bhateja N, Bhat RS, Kumar KV. Diagnostic role of platelet lymphocyte ratio(PLR) in pancreatic head masses. The Indian Journal of Surgery. 2013;75(1):4-9

[10] Kishiki T, Masaki T, Mastuoka H, Abe N, Mori T, Sugiyama M. New prognostic scoring system for incurable stage IV colorectal cancer. Asian Pacific Journal of Cancer Prevention: APJCP. 2016;17(2):597-601

[11] Buergy D, Wenz F, Groden C, Brockmann M. Tumor-platelet interaction in solid tumors. International Journal Cancer. 2012;130(12):2747-2760. DOI: 10.1002/ijc.27441

[12] Hait NC, Maiti A. The role of sphingosine-1-phosphate and ceramide-1-phosphate in inflammation and cancer. Mediators of Inflammation. 2017;2017:4806541

[13] Ikwegbue PC, Masamba P, Oyinloye BE, Kappo AP. Roles of Heat Shock Proteins in Apoptosis, Oxidative Stress, Human Inflammatory Diseases, and Cancer. Pharmaceuticals. 2018;11(1):2. DOI: 10.3390/ph11010002 
[14] Lujan DA, Ochoa JL, Hartley RS. Cold-inducible RNA binding protein in cancer and inflammation. Wiley Interdisciplinary Reviews RNA. 2018;9(2). DOI: 10.1002/wrna.1462

[15] Kakkat S, Rajan R, Sindhu RS, Natesh B, Raviram S. Comparison of platelet-lymphocyte ratio and CA 19-9 in differentiating benign from malignant head masses in patients with chronic pancreatitis. Indian Journal of Gastroenterology: Official Journal of the Indian Society of Gastroenterology. 2017;36(4):263-267

[16] Rammohan A, Cherukuri SD, Palaniappan R, Perumal SK, Sathyanesan J, Govindan M. Preoperative platelet-lymphocyte ratio augments CA 19-9 as a predictor of malignancy in chronic calcific pancreatitis. World Journal of Surgery. 2015;39(9):2323-2328

[17] Joshi RK, Lee SA. Obesity related adipokines and colorectal cancer: A review and metaanalysis. Asian Pacific Journal of Cancer Prevention: APJCP. 2014;15(1):397-405

[18] Knüpfer H, Preiss R. Serum interleukin-6 levels in colorectal cancer patients - A summary of published results. International Journal of Colorectal Disease. 2009;25(2):135-140

[19] Belluco C, Nitti D, Frantz M, Toppan P, Basso D, Plebani M, et al. Interleukin-6 blood level is associated with circulating carcinoembryonic antigen and prognosis in patients with colorectal Cancer. Annals of Surgical Oncology. 2000;7(2):133-138

[20] Ashizawa T, Okada R, Suzuki Y, Takagi M, Yamazaki T, Sumi T, et al. Clinical significance of interleukin-6 (IL-6) in the spread of gastric cancer: Role of IL-6 as a prognostic factor. Gastric Cancer. 2005;8(2):124-131

[21] Buttarello M, Mezzapelle G, Plebani M. Effect of preanalytical and analytical variables on the clinical utility of mean platelet volume. Clinical Chemistry and Laboratory Medicine. 2018;56(5):718-725. DOI: 10.1515/cclm-2017-0730

[22] Ulutas KT, Celik M. Preanalytical error occurrence rate in Central Clinic Laboratory of a Public Hospital in Turkey. Journal of Pharmacy and Pharmacology. 2016;4(1):318-321

[23] Raleigh JS, Jandrey KE, Burges J, Kent MS. Factors affecting platelet concentration in platelet concentrates from canine blood donors. Journal of Veterinary Internal Medicine. 2017;31(3):759-763

[24] Ulutas KT, Dokuyucu R, Sefil F, Yengil E, Sumbul AT, Rizaoglu H, et al. Evaluation of mean platelet volume in patients with type 2 diabetes mellitus and blood glucose regulation: A marker for atherosclerosis? International Journal of Clinical and Experimental Medicine. 2014;7(4):955-961 
